\title{
Topological methods in nonlinear analysis
}

\author{
P.J. WATSON
}

In this thesis, we investigate various topological methods for establishing the existence of solutions to nonlinear problems.

The first topic of the thesis concerns the development of a Random Degree Theory for solving nonlinear problems which depend on a parameter in a measurable way. The typical nonlinear problem which this is applicable to is of the form $L(x) \in N(\omega, x)$ where $L$ is a linear Fredholm mapping of index zero, and $N$ is a multivalued mapping, measurable with respect to $\omega$ and upper semicontinuous in $x$. Here $L: \operatorname{dom} X \rightarrow Z$ and $N: \Omega \times G \rightarrow C K(Z)$ where $\Omega$ is a measurable space, $X$ is a Banach space, $G \subset X, Z$ is a normed linear space and $C K(Z)$ denotes the set of nonempty compact convex subsets of $Z$. A solution of the above inclusion is a measurable mapping $\phi: \Omega \rightarrow X$ such that $L(\phi(\omega)) \in N(\omega, \phi(\omega))$ for almost all $\omega \in \Omega$.

The random degree has the usual properties of a degree such as homotopy invariance, excision and the important existence property. The existence property states that if the random degree is nonzero for almost all $\omega \in \Omega$, then there exists a solution of the above inclusion.

Closely related to degree theory is the theory of fixed points which is examined next. We investigate the existence of fixed points for multivalued mappings acting between a topological space which has a special convexity structure. This structure, called $H$-convexity, is analogous to the notion of convexity in vector spaces but can be defined on topological spaces without a linear structure, such as a torus or a Möbius strip. We establish an analogue of the Tychonoff fixed point theorem in this setting. A coincidence point theorem is proved and application to mathematical economics is also discussed.

The thesis concludes with several results on Variational Inequalities, Coincidence point theorems, the theory of KKM mapping (that is; Knaster-KuratowskiMazurkiewicz) and certain relationships betwen these results. In particular, in the

Received 30th June, 1998

Thesis submitted to The University of Queensland, February 1998. Degree approved, June 1998. Supervisor: Dr. Enayet U. Tarafdar.

Copyright Clearance Centre, Inc. Serial-fee code: 0004-9729/98 \$A2.00+0.00. 
setting of vector spaces, a coincidence theorem is proved and shown to be equivalent to a generalised KKM type theorem.

Department of Matehmatics

The University of Queesnland

Queensland 4072

Australia

e-mail: pjw@maths.uq.edu.au 